A $\mathrm{C} G$ Rec. Nat. Prod. 12:6 (2018) 634-637

records of natural

products

\title{
Quinolone Alkaloids Along with Other Constituents from Zanthoxylum rhetsa and their Chemotaxonomic Significance
}

\author{
Fatema-Tuz-Zohora $^{\oplus * 1,2}$, Md. Abdul Muhit ${ }^{\oplus 3}$, \\ Choudhury Mahmood Hasan ${ }^{\oplus 1}$ and Monira Ahsan ${ }^{\oplus 1}$ \\ ${ }^{1}$ Department of Pharmaceutical Chemistry, Faculty of Pharmacy, University of Dhaka, Bangladesh \\ ${ }^{2}$ Department of Pharmacy; Manarat International University, Savar, Dhaka, Bangladesh \\ ${ }^{3}$ Department of Clinical Pharmacy and Pharmacology, Faculty of Pharmacy, University of Dhaka, \\ Bangladesh
}

(Received November 18, 2017; Revised February 02, 2018; Accepted February 14, 2018;)

\begin{abstract}
A total of eight compounds were isolated from the petroleum ether and chloroform extracts of the root bark of Zanthoxylum rhetsa (Roxb.) (D.C). These are a coumarin xanthyletin (1), five quinolone alkaloids zanthodioline (2), arnottianamide (3), skimmianine (4), fagaridine (5), oxynitidine (7), a lignan pluviatilol (6), and a triterpene lupeol (8) were identified. Structures of the isolates were characterized by extensive 1D and 2D NMR analyses and by comparing their spectral data with the published values. The compounds, zanthodioline (2), oxynitidine (7), fagaridine (5), and pluviatilol (6) are the first record from this plant.
\end{abstract}

Keywords: Zanthoxylum rhetsa; quinolone alkaloids; lignan; triterpene; NMR spectroscopy. ㄷ 2018 ACG Publications. All rights reserved.

\section{Plant Source}

The root barks of Zanthoxylum rhetsa were collected from Narsingdi disrict, Bangladesh in the month of August, 2013. The plant part was identified by a taxonomist (Ms. Nasrin Aktar), Bangladesh National Herbarium where a voucher specimen was deposited for future reference (DACB Accession no. 42528).

\section{Previous Studies}

A series of isoquinoline and quinoline alkaloids, including canthine-6-one, 8-methoxy-Nmethylfindersine, dihydrochelerythrine, chelerythrine, rhetine, rhetsine, rhetsinine, dihydroavicine, dictamnine, arborine and others have been isolated from the plant [1].

\footnotetext{
* Corresponding author: E- Mail: fatema.zohora41@gmail.com (F. T. Zohora), Phone +88-01675338774.
} 


\section{Present Study}

The air dried and powdered root barks of Z. rhetsa $(3.5 \mathrm{~kg})$ were extracted with methanol over the period of 15 days. The crude methanol extract $(40 \mathrm{~g})$ was then fractionated sequentially by petroleum ether $(9 \mathrm{~g})$, ethyl acetate $(6 \mathrm{~g})$, chloroform $(12 \mathrm{~g})$ and methanol $(12 \mathrm{~g})$ fractions with continuous stirring. Petroleum ether soluble fraction was subjected to silica gel column and was fractionated with a gradient of petroleum ether-dichloromethane- ethyl acetate-methanol which was given total of 551 fractions each with $20 \mathrm{ml}$. After TLC screening, similar fractions were mixed together and purified the compounds by recrystallization afforded xanthyletin $(\mathbf{1}, 100.6 \mathrm{mg})$, zanthodioline $(\mathbf{2}, 50.1 \mathrm{mg})$ and arnottianamide $(\mathbf{3}, 20 \mathrm{mg})$. Similarly the chloroform soluble fraction was loaded over silica gel column chromatography and fractionated with a solvent system of increasing polarity and yielding skimmianine $(4,200.4 \mathrm{mg})$. Similar fractions of silica column were mixed and further fractionated with sephadex LH-20 gel column eluted with petroleum etherchloroform (20:80) to yield fagaridine $(5,2.5 \mathrm{mg})$, pluviatilol $(\mathbf{6}, 6 \mathrm{mg})$ and oxynitidine $(7,5.3 \mathrm{mg})$. In the current investigation, a total of eight compounds have been isolated from the root bark of Zanthoxylum rhetsa (Roxb.) (D.C). among these, zanthodioline (2), fagaridine (5), pluviatilol (6) and oxynitidine (7) are the first time report from this species.

Zanthodioline (2): white crystals; ${ }^{1} \mathrm{H}-\mathrm{NMR}\left(500 \mathrm{MHz}, \mathrm{CDCl}_{3}\right): \delta 7.64$ (dd, $\left.J=8.0,1.2 \mathrm{~Hz}, \mathrm{H}-5\right), 7.22$ $(1 \mathrm{H}, \mathrm{t}, J=8.0 \mathrm{~Hz}, \mathrm{H}-6), 7.12(1 \mathrm{H}, \mathrm{dd}, J=8.0,1.2 \mathrm{~Hz}, \mathrm{H}-7), 4.76\left(1 \mathrm{H}, \mathrm{d}, J=8 \mathrm{~Hz}, \mathrm{H}-4^{\prime}\right), 3.97(3 \mathrm{H}, \mathrm{s}$, $\mathrm{N}-\mathrm{Me}), 3.93$ (3H, s, OMe-8), $3.84\left(1 \mathrm{H}, \mathrm{d}, J=8 \mathrm{~Hz}, \mathrm{H}-3^{\prime}\right), 1.64\left(3 \mathrm{H} \mathrm{s}, \mathrm{H}-2^{\prime} \mathrm{Me}\right), 1.33\left(3 \mathrm{H}, \mathrm{s}, \mathrm{H}-2^{\prime} \mathrm{Me}\right)$. ${ }^{13} \mathrm{C}-\mathrm{NMR}\left(125 \mathrm{MHz}, \mathrm{CDCl}_{3}\right): \delta 164.6(\mathrm{C}-2), 154.8(\mathrm{C}-4), 148.8(\mathrm{C}-8), 130.8(\mathrm{C}-9), 122.8(\mathrm{C}-6), 118.2$ (C-10), 116.2 (C-5), 114.4 (C-7), 106.0 (C-3), 80.9 (C-2'), 75.3 (C-3'), 67.7 (C-4'), 56.7 (OMe-8), 34.8 (N-Me), 26.1 (C-2' Me), 19.3 (C-2' Me).

Fagaridine (5): light yellow powder; ${ }^{1} \mathrm{H}-\mathrm{NMR}\left(500 \mathrm{MHz}, \mathrm{CDCl}_{3}\right): \delta 9.71(1 \mathrm{H}, \mathrm{s}, \mathrm{H}-8), 8.67(1 \mathrm{H}, \mathrm{d}, J=$ $8.8 \mathrm{~Hz}, \mathrm{H}-12), 8.31(1 \mathrm{H}, \mathrm{s}, \mathrm{H}-1), 8.24(1 \mathrm{H}, \mathrm{d}, J=9.2 \mathrm{~Hz}, \mathrm{H}-5), 7.99(1 \mathrm{H}, \mathrm{d}, J=9.2 \mathrm{~Hz}, \mathrm{H}-11), 7.57$ $(1 \mathrm{H}, \mathrm{s}, \mathrm{H}-4), 7.54(1 \mathrm{H}, \mathrm{d}, J=8.8 \mathrm{~Hz}, \mathrm{H}-6), 6.23\left(2 \mathrm{H}, \mathrm{s}, \mathrm{OCH}_{2} \mathrm{O}\right), 5.50(3 \mathrm{H}, \mathrm{s}, \mathrm{N}-\mathrm{Me}), 4.28(3 \mathrm{H}, \mathrm{s}$, OMe-9).

Pluviatilol (6): white crystals; ${ }^{1} \mathrm{H}-\mathrm{NMR}\left(500 \mathrm{MHz}, \mathrm{CDCl}_{3}\right): \delta 6.97(1 \mathrm{H}, \mathrm{d}, J=1.2 \mathrm{~Hz}, \mathrm{H}-2), 6.92(1 \mathrm{H}$, $\mathrm{d}, J=8.0 \mathrm{~Hz}, \mathrm{H}-5), 6.89\left(1 \mathrm{H}, \mathrm{d}, J=1.2 \mathrm{~Hz}, \mathrm{H}-2^{\prime}\right), 6.85\left(1 \mathrm{H}, \mathrm{dd}, J=8.0,1.2 \mathrm{~Hz}, \mathrm{H}-6^{\prime}\right), 6.81(1 \mathrm{H}, \mathrm{dd}$, $J=8.0,2.0 \mathrm{~Hz}, \mathrm{H}-6), 6.80\left(1 \mathrm{H}, \mathrm{d}, J=8.0 \mathrm{~Hz}, \mathrm{H}-5^{\prime}\right), 5.98\left(2 \mathrm{H}, \mathrm{s}, \mathrm{OCH}_{2} \mathrm{O}\right), 4.87(1 \mathrm{H}, \mathrm{d}, J=5.2 \mathrm{~Hz}, \mathrm{H}-7)$, $4.44\left(1 \mathrm{H}, \mathrm{d}, J=6.8 \mathrm{~Hz}, \mathrm{H}-7^{\prime}\right), 4.13\left(1 \mathrm{H}, \mathrm{d}, J=9.6 \mathrm{~Hz}, \mathrm{H}^{\prime} 9^{\prime} \alpha\right), 3.93(3 \mathrm{H}, \mathrm{s}, \mathrm{OMe}-3), 3.87(1 \mathrm{H}, \mathrm{dd}, J=$ 9.6, $6.0 \mathrm{~Hz}, \mathrm{H}-9 \alpha), 3.86\left(1 \mathrm{H}, \mathrm{m}, \mathrm{H}-9^{\prime} \beta\right), 3.34(1 \mathrm{H}, \mathrm{m}, \mathrm{H}-8), 3.34(1 \mathrm{H}, \mathrm{m}, \mathrm{H}-9 \beta), 2.89$ (1H, dd, $J=7.2$, $\left.6.4 \mathrm{~Hz}, \mathrm{H}-8^{\prime}\right) .{ }^{13} \mathrm{C}-\mathrm{NMR}\left(125 \mathrm{MHz}, \mathrm{CDCl}_{3}\right): \delta 148.0^{*}\left(\mathrm{C}-4^{\prime}\right), 147.2^{*}\left(\mathrm{C}-3^{\prime}\right), 145.9^{*}(\mathrm{C}-3), 144.7^{*}(\mathrm{C}-$ 4), 130.3 (C-1), 119.5 (C-6'), 118.4 (C-6), 114.2 (C-5), 108.4 (C-2), 108.2 (C-5'), 106.5 (C-2'), 101.0 $\left(\mathrm{OCH}_{2} \mathrm{O}\right), 87.7\left(\mathrm{C}-7^{\prime}\right), 82.1(\mathrm{C}-7), 71.0\left(\mathrm{C}-9^{\prime}\right), 69.8(\mathrm{C}-9), 56.0(\mathrm{OMe}-3), 54.6\left(\mathrm{C}-8^{\prime}\right), 50.2(\mathrm{C}-8)$.

Oxynitidine (7): white amorphous; ${ }^{1} \mathrm{H}-\mathrm{NMR}\left(500 \mathrm{MHz}, \mathrm{CDCl}_{3}\right): \delta 8.02(1 \mathrm{H}, \mathrm{d}, J=8.8 \mathrm{~Hz}, \mathrm{H}-6), 7.96$ $(1 \mathrm{H}, \mathrm{s}, \mathrm{H}-9), 7.68(1 \mathrm{H}, \mathrm{s}, \mathrm{H}-12), 7.63(1 \mathrm{H}, \mathrm{s}, \mathrm{H}-1), 7.60(1 \mathrm{H}, \mathrm{d}, J=8.8 \mathrm{~Hz}, \mathrm{H}-5), 7.22(1 \mathrm{H}, \mathrm{s}, \mathrm{H}-4)$, $6.13\left(2 \mathrm{H}, \mathrm{s}, \mathrm{OCH}_{2} \mathrm{O}\right), 4.14(3 \mathrm{H}, \mathrm{s}, \mathrm{OMe}-11), 4.08(3 \mathrm{H}, \mathrm{s}, \mathrm{OMe}-11), 4.02(3 \mathrm{H}, \mathrm{s}, \mathrm{N}-\mathrm{Me})$. 
<smiles>CC1(C)C=Cc2cc3ccc(=O)oc3cc2O1</smiles><smiles>COc1cccc2c3c(c(=O)n(C)c12)[C@H](O)[C@H](O)C(C)(C)O3</smiles><smiles>COc1ccc(-c2ccc3cc4c(cc3c2N(C)C=O)OCO4)c(O)c1OC</smiles><smiles>CCCCCCC</smiles><smiles>COc1ccc2c(OC)c3ccoc3nc2c1OC</smiles>

$\mathrm{OCH}_{3}$<smiles>COc1cc([C@H]2OC[C@@H]3[C@@H](c4ccc5c(c4)OCO5)OC[C@H]23)ccc1O</smiles><smiles>COc1cc2c(=O)n(C)c3c4cc5c(cc4ccc3c2cc1OC)OCO5</smiles>

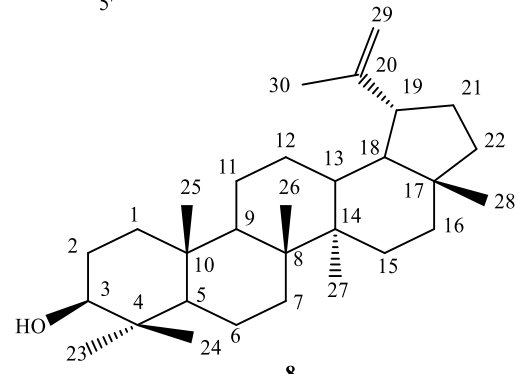

Figure 1. Compounds 1-8 isolated from Z. rhetsa

\section{Chemotaxonomic Significance}

In our present investigation out of eight compounds, four namely zanthodioline (2), fagaridine (5), pluviatilol (6) and oxynitidine (7) are the first report from this species. The pyranoquinoline alkaloid Zanthodioline (2) was previously isolated from Zanthoxylum simulans [2]. The benzophenanthridine alkaloids fargaridine (5) and oxynitidine (7) have been reported respectively from Zanthoxylum rhoifolium [3] and Zanthoxylum nitidum [4]. The benzophenanthridine alkaloids are the most commonly found in the genus [5]. The isolation of the lignan pluviatilol (6) earlier reported from Zanthoxylum acanthopodium [6] and Zanthoxylum pluviatile [7] also support a close relationship among these three rutaceous species.

\section{Acknowledgments}

The authors are thankful to Professor Dr. Kaoru Umehara, School of Pharmaceutical Sciences, University of Shizuoka, Japan for his support for taking NMR spectra of the isolated compounds.

\section{Supporting Information}

Supporting Information accompanies this paper on http://www.acgpubs.org/RNP 


\section{ORCID}

Fatema-Tuz-Zohora: 0000-0002-6087-0425

Md. Abdul Muhit: 0000-0002-5958-9935

Choudhury Mahmood Hasan: 0000-0001-7026-4368

Monira Ahsan: 0000-0002-1326-9063

\section{References}

[1] M. Ahsan, M.R. Haque, M.B. Hossain, S.K. Islam, A.I. Gray and C.M. Hasan (2014). Cytotoxic dimeric quinolone-terpene alkaloids from the root bark of Xanthoxylum rhetsa, Phytochemistry 103, 8-12.

[2] I.S. Chen, I.W. Tsai, C.M. Teng, J.J. Chen, Y.L. Chang, F.N. Ko, M.C. Lu and J.M. Pezzuto (1997). Pyranoquinoline Alkaloids from Zanthoxylum simulans, Phytochemistry 46, 525-529.

[3] L.O.J. Patino, R.J.A. Prieto and S.L.E. Cuca (2012). Bioactive compounds in Phytomedicine, InTech publishers, London, United Kingdom, p. 205.

[4] H.R. Arthur, W.H. Hui, and Y.L. Ng (1959). An examination of the rutaceae of Hong Kong. Part II. The alkaloids, nitidine and oxynitidine, from Zanthoxylum nitidum, J. Chem. Soc. 0, 1840-1845.

[5] B.D. Krane, M.O. Fagbule, M. Shamma and B. Gozler (1984). The Benzophenanthridine Alkaloids, J. Nat. Prod., 47, 1-43.

[6] H.G. Choi, Y.H. Choi, J.H. Kim, H.H. Kim, S.H. Kim, J.A. Kim, S.M. Lee, M. Na and S.H. Lee (2014). A new neolignan and lignans from the stems of Lindera obtusiloba Blume and their anti-allergic inflammatory effects, Arch. Pharm. Res., 37, 467-472.

[7] V.N. Pandey, S.C. Malhotra, D.P. Sharma and J.P. Kotiyal (1990). Katipaya Ayurvediya Vanaspatiyom Ka Padapa Rasayanika Parikshana. Central Council for Research in Ayurveda \& Siddha, Ministry of Health and Family Welfare, Government of India, p. 336-337.

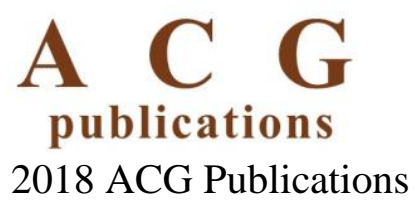

